\title{
On the Néron-Severi lattice of a Delsarte surface
}

\author{
Alex Degtyarev
}

\begin{abstract}
We suggest an algorithm computing, in some cases, an explicit generating set for the Néron-Severi lattice of a Delsarte surface. In a few special cases, including those of Fermat surfaces and cyclic Delsarte surfaces that were previously conjectured in the literature, we show that certain "obvious" divisors do generate the lattice. The proof is based on the computation of the Alexander module related to a certain abelian covering.
\end{abstract}

\section{Introduction}

Throughout the article, all algebraic varieties are over $\mathbb{C}$.

\subsection{Statement of the problem}

A Delsarte surface is a surface $\Phi_{A} \subset \mathbb{P}^{3}$ given by a four-term equation of the form (see [3], [8])

$$
\sum_{i=0}^{3} \prod_{j=0}^{3} z_{j}^{a_{i j}}=0 .
$$

The restrictions to the matrix $A:=\left[a_{i j}\right]$ are listed in Section 2.2 as items (1)-(4).

We are interested in certain birational invariants of Delsarte surfaces. For this reason, we silently replace $\Phi_{A}$ with its resolution of singularities. The particular choice of the resolution is not important; for example, one can take the minimal one.

For an alternative description of Delsarte surfaces, introduce the multiplicative abelian group $\mathbb{G} \cong \mathbb{Z}^{3}$ with a distinguished generating set $t_{0}, t_{1}, t_{2}, t_{3}$ subject to the only relation $t_{0} t_{1} t_{2} t_{3}=1$. Then, each epimorphism $\alpha$ : $\mathbb{G} \rightarrow G$ to a finite group $G$ gives rise to a Delsarte surface $\Phi[\alpha]$ (see Sections 2.2 and 2.2). By an abuse of language, an epimorphism $\alpha$ as above is referred to as a finite quotient of $\mathbb{G}$.

\section{DEFINITION 1.2}

In the examples, we will consider the following four special classes of Delsarte surfaces, corresponding to special finite quotients $\alpha: \mathbb{G} \rightarrow G$ : 
(1) Fermat surfaces $\Phi[m]$, where an integer $m \in \mathbb{N}_{+}$is regarded as the quotient projection $m: \mathbb{G} \rightarrow \mathbb{G} / m \mathbb{G}$;

(2) unramified (at $\infty$ ) Delsarte surfaces $\Phi[\alpha]$, that is, such that $\alpha\left(t_{0}\right)=1$;

(3) cyclic Delsarte surfaces $\Phi[\alpha]$, that is, such that $G$ is a cyclic group;

(4) diagonal Delsarte surfaces $\Phi[\mathfrak{m}]$, where a vector $\mathfrak{m}:=\left(m_{1}, m_{2}, m_{3}\right) \in \mathbb{N}_{+}^{3}$ is regarded as the quotient projection $\mathfrak{m}: \mathbb{G} \rightarrow \mathbb{G} /\left(t_{1}^{m_{1}}=t_{2}^{m_{2}}=t_{3}^{m_{3}}=1\right)$.

(To avoid the common confusion, we use $\mathbb{N}_{+}$for the set of positive integers.) Note that, in items (2) and (4), the definition depends on the order of the indices, and we relate a surface $\Phi[\alpha]$ to the corresponding class whenever it satisfies the condition after a possible permutation of the indices $(0,1,2,3)$.

By Poincaré duality, the Néron-Severi lattice $\mathrm{NS}(\Phi[\alpha])$ can be regarded as a subgroup of the homology group $H_{2}(\Phi[\alpha]) /$ Tors. Our primary interest is the extent to which $\operatorname{NS}(\Phi[\alpha])$ is generated by the components of a certain "obvious" divisor $V[\alpha] \subset \Phi[\alpha]$ (see Section 2.3). (In the case of Fermat surfaces, this divisor $V$ is essentially constituted by the lines contained in the surface.) To this end, we consider the homomorphism $\iota_{*}: H_{2}(V[\alpha]) \rightarrow \mathrm{NS}(\Phi[\alpha])$ induced by the inclusion $\iota: V[\alpha] \hookrightarrow \Phi[\alpha]$ and introduce the groups

$$
\mathbf{S}[\alpha]:=\operatorname{Im} \iota_{*}, \quad \mathbf{K}[\alpha]:=\operatorname{Ker} \iota_{*}, \quad \mathbf{T}[\alpha]:=\operatorname{Tors}(\operatorname{NS}(\Phi[\alpha]) / \mathbf{S}[\alpha]) .
$$

We compute the two latter groups, which are birational invariants of the surface.

The motivation for our interest is Shioda's [8] algorithm computing the Picard rank $\rho(\Phi[\alpha]$ ). In some cases (most notably, if $|G|$ is prime to 6; cf. Corollary 1.8 below), this computation implies that $\mathrm{NS}(\Phi[\alpha]) \otimes \mathbb{Q}=\mathbf{S}[\alpha] \otimes \mathbb{Q}$, that is, $\operatorname{NS}(\Phi[\alpha])$ is generated by the components of $V[\alpha]$ over $\mathbb{Q}$. Hence, a natural question is if this generation property still holds over the integers, that is, if $\mathbf{T}[\alpha]=0$. We answer this question in the affirmative for a few special classes of surfaces, while showing that, in general, the answer is in the negative.

\subsection{Principal results}

We introduce the following subgroups of $\mathbb{G}$ :

- $\mathbb{G}_{i j}$ is generated by $t_{i}$ and $t_{j}, i, j=0,1,2,3$;

- $\mathbb{G}_{i}$ is generated by $t_{i} t_{j}$ and $t_{i} t_{k}, i=1,2,3$ and $\{i, j, k\}=\{1,2,3\}$;

- $\mathbb{G}_{=}:=\sum_{i} \mathbb{G}_{i}$ is generated by $t_{1} t_{2}, t_{1} t_{3}$, and $t_{2} t_{3}$.

Given a finite quotient $\alpha: \mathbb{G} \rightarrow G$, denote $G_{*}:=G / \alpha\left(\mathbb{G}_{*}\right)$ (for a subscript $*$ of the form $i j, i$, or $=$ ), and let $\delta[\alpha]:=\left|G_{=}\right|-1 \in\{0,1\}$. (In more symmetric terms, $\mathbb{G}_{i}$ depends only on the partition $\{0, i\} \cup\{j, k\}$ of the index set, and $\mathbb{G}=$ is generated by all products $t_{i} t_{j}, i, j=0,1,2,3$; one has $[\mathbb{G}: \mathbb{G}=]=2$.)

Recall that the length $\ell(A)$ of a finitely generated abelian group $A$ is the minimal number of generators of $A$, and the exponent $\exp A$ of a finite abelian group $A$ is the minimal positive integer $m$ such that $m A=0$. For a finite quotient $\alpha: \mathbb{G} \rightarrow G$, the $\operatorname{exponent} \exp G$ is the minimal positive integer $m$ such that $m \mathbb{G} \subset \operatorname{Ker} \alpha$, and we can also define the height ht $\alpha:=\exp G / n$, where $n$ is the 
maximal integer such that $\operatorname{Ker} \alpha \subset n \mathbb{G}$. Note that $(\exp G)^{3} /|G|$ is an integer dividing $(\text { ht } \alpha)^{2}$.

The principal results of the article (combined with those of [2]) are stated below, with references to the proofs given in the statements.

\section{THEOREM 1.4 (SEE [2] AND SECTION 3.1)}

For any finite quotient $\alpha: \mathbb{G} \rightarrow G$, one has

$$
\pi_{1}(\Phi[\alpha])=H_{1}(\Phi[\alpha])=\operatorname{Ker} \alpha / \prod\left(\mathbb{G}_{i j} \cap \operatorname{Ker} \alpha\right),
$$

the product running over all pairs $0 \leq i<j \leq 3$. This group is trivial for any of the four special classes of Delsarte surfaces introduced in Definition 1.2. In general, the group $\pi_{1}(\Phi[\alpha])$ is cyclic and its order $\left|\pi_{1}(\Phi[\alpha])\right|$ divides ht $\alpha$.

\section{THEOREM 1.5 (SEE SECTION 4.2)}

For any finite quotient $\alpha: \mathbb{G} \rightarrow G$, one has

$$
\operatorname{rk} \mathbf{K}[\alpha]=\sum_{0 \leq i<j \leq 3}\left|G_{i j}\right|+\sum_{1 \leq i \leq 3}\left|G_{i}\right|-3-\delta[\alpha] .
$$

Besides, one has $\ell(\mathbf{T}[\alpha]) \leq 6+\delta[\alpha]$ and $\exp \mathbf{T}[\alpha]$ divides $(\exp G)^{3} /|G|$.

\section{ADDENDUM 1.6 (SEE SECTION 4.4)}

As a lattice, $\mathbf{S}[\alpha]=H_{2}(V[\alpha]) /$ ker, where ker is the kernel ker $H_{2}(V[\alpha])$ of the intersection index form.

Note that Theorem 1.5 is merely an estimate on the size of the torsion $\mathbf{T}[\alpha]$, most interesting being the fact that the length of this group is universally bounded. A better estimate is found in Lemma 4.8, and a precise, although not very efficient, algorithm for computing this group is given by (3.8). A few examples, showing the sharpness of most estimates, are considered in Section 6. It appears that there should be better bounds taking into account the group $\pi_{1}(\Phi[\alpha]$ ) (see Remark 6.2).

Note also that the rank formula in Theorem 1.5 states, essentially, that the rank $\operatorname{rk} \mathbf{K}[\alpha]$ is the "minimal possible." More precisely, $G$ acts on $\Phi[\alpha]$ and $V[\alpha]$ (see Section 2.2), and the space $H_{2}(\Phi[\alpha] ; \mathbb{C})$ splits into multi-eigenspaces, which are all of dimension at most 1 (see [7], [8]). Comparing the dimensions (or using the explicit description of the kernel; see Section 4.2), one can see that each eigenspace present in $H_{2}(V[\alpha] ; \mathbb{C})$ is mapped epimorphically onto the corresponding eigenspace in $H_{2}(\Phi[\alpha] ; \mathbb{C})$.

\section{THEOREM 1.7}

One has $\mathbf{T}[\alpha]=0$ in each of the following three cases:

(1) Fermat surfaces $\Phi[\alpha], \alpha=m \in \mathbb{N}_{+}$(see [2] or Section 4.1);

(2) Delsarte surfaces unramified at $\infty$ (see [2]);

(3) cyclic Delsarte surfaces (see Section 5).

Besides, one has the following stronger bound: 
(4) if $\Phi[\alpha]$ is diagonal, $\alpha=\left(m_{1}, m_{2}, m_{3}\right) \in \mathbb{N}_{+}^{3}$, then $\ell(\mathbf{T}[\alpha]) \leq \delta[\alpha]$ and the order $|\mathbf{T}[\alpha]|$ divides $\operatorname{lcm}_{1 \leq i<j \leq 3}\left(\operatorname{gcd}\left(m_{i}, m_{j}\right)\right) / \operatorname{gcd}\left(m_{1}, m_{2}, m_{3}\right)$ (see Section 4.3).

For Fermat surfaces, the primitivity statement was suggested in [7] and [1], and it was verified numerically in [5] for all values of $m$ prime to 6 in the range $5 \leq m \leq 100$. For cyclic Delsarte surfaces (Theorem 1.7(3)), the statement was conjectured in [6], where it was verified for all cyclic quotients $\alpha: \mathbb{G} \rightarrow G$ with $|G| \leq 50$.

\section{COROLLARY 1.8 (SEE SECTION 4.5)}

Let $\alpha: \mathbb{G} \rightarrow G$ be a finite quotient with $\Phi[\alpha]$ in one of the four special classes introduced in Definition 1.2, and assume that $|G|$ is prime to 6 . Then the group $\operatorname{Pic} \Phi[\alpha]=\operatorname{NS}(\Phi[\alpha])$ is generated by the components of $V[\alpha]$. In other words, $\mathrm{NS}(\Phi[\alpha])=H_{2}(V[\alpha]) /$ ker as a lattice (see Addendum 1.6).

It is worth emphasizing that, since both the action of $G$ (obvious) and the intersection matrix of $V[\alpha]$ (see, e.g., [5]) are known, Corollary 1.8 gives us a complete description of the Néron-Severi group $\operatorname{NS}(\Phi[\alpha])$, including the lattice structure and the action of $G \subset$ Aut $\Phi[\alpha]$. In general, if $\operatorname{NS}(\Phi[\alpha]) \otimes \mathbb{Q}=\mathbf{S}[\alpha] \otimes \mathbb{Q}$ but $\mathbf{T}[\alpha] \neq 0$, then the lattice structure can be recovered using the algorithm outlined in Section 3.4 .

\subsection{Contents of the article}

In Section 2, we introduce Delsarte surfaces $\Phi$ and the "obvious" divisors $V \subset$ $\Phi$ and discuss their description in terms of ramified coverings of the plane. In Section 3, most principal results of the article are reduced to the problem of analyzing the integral torsion of a certain Alexander module (see (3.8) and (3.9)). Most results are proved in Section 4; an exception is the case of cyclic Delsarte surfaces, which is treated separately (and slightly differently) in Section 5. Finally, in Section 6, we discuss a few numeric examples (obtained from experiments with small random matrices), illustrating the sharpness of most bounds on the one hand and the complexity of the general problem on the other.

\section{Preliminaries}

\subsection{Conventions}

The notation Tors $A$ stands for the $\mathbb{Z}$-torsion of an abelian group $A$. We emphasize that Tors always refers to the integral torsion, even if $A$ is a module over a larger ring. This convention applies also to the rank $\mathrm{rk} A$ and length $\ell(A)$ : we regard $A$ as an abelian group. We abbreviate $A /$ Tors $:=A /$ Tors $A$.

We denote by $\psi_{m}(t)$ the cyclotomic polynomial of order $m$, that is, the irreducible (over $\mathbb{Q}$ ) factor of $t^{m}-1$ that does not divide $t^{n}-1$ for $1 \leq n<m$. We also make use of the polynomials $\tilde{\varphi}_{m}(t):=\left(t^{m}-1\right) /(t-1), m \in \mathbb{N}_{+}$. 
Unless stated otherwise, all homology and cohomology groups have coefficients in $\mathbb{Z}$. Since all spaces involved have homotopy type of $C W$-complexes, the choice of a theory is not important; for example, one can use singular (co-) homology.

Given a closed oriented 4-manifold $X$, we identify $H^{2}(X)=H_{2}(X)$ by means of Poincaré duality. In particular, if $X$ is a smooth compact complex analytic surface, we regard the Néron-Severi lattice $\mathrm{NS}(X)$ as a sublattice of $H_{2}(X) /$ Tors (with the usual intersection index pairing), so that a divisor $D \subset X$ is represented by its (topological) fundamental class $[D] \in H_{2}(X) /$ Tors.

Consider a smooth compact analytic surface $X$ and a divisor $D \subset X$. In what follows, we are only interested in the (reduced) irreducible components of $D$ and its support; hence, without loss of generality, we can assume $D$ effective and reduced, using the same notation for the support of $D$. Given $X$ and a $D \subset X$ as above, we denote by $\mathbf{S}\langle D\rangle \subset \mathrm{NS}(X)$ the subgroup generated by the irreducible components of $D$. In other words,

$$
\mathbf{S}\langle D\rangle=\operatorname{Im}\left[\iota_{*}: H_{2}(D) \rightarrow H_{2}(X) / \text { Tors }\right],
$$

where $\iota: D \hookrightarrow X$ is the inclusion. We will also consider the groups

$$
\mathbf{T}\langle D\rangle:=\operatorname{Tors}(\mathrm{NS}(X) / \mathbf{S}\langle D\rangle), \quad \mathbf{K}\langle D\rangle:=\operatorname{Ker}\left[\iota_{*}: H_{2}(D) \rightarrow H_{2}(X) / \text { Tors }\right],
$$

which are birational invariants of the pair $(X, D)$. More precisely, if $\sigma: X^{\prime} \rightarrow X$ is a blowdown map and $D^{\prime}:=\sigma^{*} D$, then $\sigma^{*}$ and $\sigma_{*}$ induce isomorphisms

$$
\mathrm{NS}\left(X^{\prime}\right) / \mathbf{S}\left\langle D^{\prime}\right\rangle=\mathrm{NS}(X) / \mathbf{S}\langle D\rangle, \quad \mathbf{T}\left\langle D^{\prime}\right\rangle=\mathbf{T}\langle D\rangle, \quad \mathbf{K}\left\langle D^{\prime}\right\rangle=\mathbf{K}\langle D\rangle .
$$

\subsection{Delsarte surfaces (see [8])}

Consider the surface $\Phi_{A}$ given by (1.1), where the exponent matrix $A:=\left[a_{i j}\right]$ is assumed to satisfy the following conditions:

(1) each entry $a_{i j}, 0 \leq i, j \leq 3$, is a nonnegative integer;

(2) each column of $A$ has at least one zero;

(3) $(1,1,1,1)^{t}$ is an eigenvector of $A$, that is, $\sum_{j=0}^{3} a_{i j}=\lambda=\operatorname{const}(i)$;

(4) $A$ is nondegenerate, that is, $\operatorname{det} A \neq 0$.

Condition (2) asserts that the surface does not contain a coordinate plane, and (3) makes (1.1) homogeneous, the degree being the eigenvalue $\lambda$.

Following [8], introduce the cofactor matrix $A^{*}:=(\operatorname{det} A) A^{-1}$, and let

$$
d:=\operatorname{gcd}\left(a_{i j}^{*}\right), \quad m:=|\operatorname{det} A| / d, \quad B=\left[b_{i j}\right]:=m A^{-1}= \pm d^{-1} A^{*} .
$$

Denoting by $\Phi[m]$ the Fermat surface $\left\{z_{0}^{m}+\cdots+z_{3}^{m}=0\right\}$, we have maps

$$
\Phi[m] \stackrel{\pi_{B}}{\longrightarrow} \Phi_{A} \stackrel{\pi_{A}}{\longrightarrow} \Phi:=\Phi[1]
$$

given by

$$
\pi_{B}:\left(z_{i}\right) \mapsto\left(\prod_{j=0}^{3} z_{j}^{b_{i j}}\right), \quad \pi_{A}:\left(z_{i}\right) \mapsto\left(\prod_{j=0}^{3} z_{j}^{a_{i j}}\right)
$$


Both maps are ramified coverings; $\pi_{A}$ and $\pi_{B} \circ \pi_{A}:\left(z_{i}\right) \mapsto\left(z_{i}^{m}\right)$ are ramified over the union $R:=R_{0}+R_{1}+R_{2}+R_{3} \subset \Phi$ of the traces of the coordinate planes, $R_{i}:=\Phi \cap\left\{z_{i}=0\right\}$.

The fundamental group $\pi_{1}(\Phi \backslash R)$ is abelian, and by Poincaré-Lefschetz duality, there are canonical isomorphisms

$$
\pi_{1}(\Phi \backslash R)=H^{2}(R) / H^{2}(\Phi)=\mathbb{G},
$$

where $\mathbb{G}$ is the abelian group introduced in Section 1.1 and a generator $t_{i} \in$ $\mathbb{G}$ evaluates to the Kronecker symbol $\delta_{i j}$ on the fundamental class $\left[R_{j}\right]$ (with its canonical complex orientation). Thus, away from the ramification locus $R$, the unramified topological covering $\pi_{A}$ is uniquely determined by a finite index subgroup of $\mathbb{G}$, namely, the image of $\pi_{1}\left(\Phi_{A} \backslash \pi_{A}^{-1}(R)\right)$, or, equivalently, by a finite quotient $\alpha: \mathbb{G} \rightarrow G$.

Due to condition (3) above, $A$ and $B$ can be regarded as endomorphisms of $\mathbb{G}$, inducing endomorphisms $A_{m}, B_{m}: \mathbb{G} / m \mathbb{G} \rightarrow \mathbb{G} / m \mathbb{G}$. Obviously, one has

$$
m \mathbb{G} \subset \operatorname{Ker} \alpha, \quad \Gamma:=\operatorname{Ker} \alpha / m \mathbb{G}=\operatorname{Ker} B_{m}=\operatorname{Im} A_{m}, \quad \operatorname{Im} B_{m}=\operatorname{Ker} A_{m},
$$

and $\Phi_{A}$ is birationally isomorphic to $\Phi[m] / \Gamma$, where a generator $t_{i} \in \mathbb{G} / m \mathbb{G}$ acts on $\Phi[m]$ by multiplying the $i$ th coordinate by a fixed primitive $m$ th root of unity.

Summarizing, we can disregard the original exponent matrix $A$ and (1.1) and adopt the following definition (cf. [6]).

\section{DEFINITION 2.2}

Given a finite quotient $\alpha: \mathbb{G} \rightarrow G$, the Delsarte surface $\Phi[\alpha]$ is defined as (any) smooth analytic compactification of the (unramified) covering of the complement $\Phi \backslash R$ corresponding to $\alpha$.

Since the invariants in which we are interested are of a birational nature (cf. (2.1)), the particular choice of the compactification in Definition 2.2 is not important. It is fairly obvious that any surface $\Phi[\alpha]$ is a resolution of singularities of the projective surface given by an appropriate equation (1.1); however, we do not use this fact. For the covering Fermat surface $\Phi[m]$, we can merely take $m=\exp G$ or any multiple thereof, so that $m \mathbb{G} \subset \operatorname{Ker} \alpha$.

\subsection{The divisor $V[\alpha]$}

Fix a finite quotient $\alpha: \mathbb{G} \rightarrow G$, and let $\pi: \Phi[\alpha] \rightarrow \Phi$ be the covering projection. Consider the lines $L_{i}:=\Phi \cap\left\{z_{0}+z_{i}=0\right\}, i=1,2,3$, let $L:=L_{1}+L_{2}+L_{3}$, and define the divisors

$$
R_{*}[\alpha]:=\pi^{*} R_{*}, \quad L_{*}[\alpha]:=\pi^{*} L_{*}, \quad V[\alpha]:=R[\alpha]+L[\alpha]
$$

on $\Phi[\alpha]$. (Here, the subscript $*$ is either empty or an appropriate index in the range $0, \ldots, 3$.) To avoid excessive nested parentheses, we introduce the shortcuts (cf. $(1.3)$ )

$$
\mathbf{S}[\alpha]:=\mathbf{S}\langle V[\alpha]\rangle, \quad \mathbf{K}[\alpha]:=\mathbf{K}\langle V[\alpha]\rangle, \quad \mathbf{T}[\alpha]:=\mathbf{T}\langle V[\alpha]\rangle,
$$


and let $\Phi^{\circ}[\alpha]:=\Phi[\alpha] \backslash V[\alpha]$. We recall that the pullback of each $L_{i}, i=1,2,3$, in the covering Fermat surface $\Phi[m]$ splits into $m^{2}$ obvious straight lines, namely,

$$
\begin{array}{ll}
L_{1}(\zeta, \eta): & (r: \omega \zeta r: s: \omega \eta s), \\
L_{2}(\zeta, \eta): & (r: s: \omega \zeta r: \omega \eta s), \\
L_{3}(\zeta, \eta): & (r: s: \omega \eta s: \omega \zeta r),
\end{array}
$$

where $(\zeta, \eta)$ is a pair of $m$ th roots of unity (parameterizing the $m^{2}$ lines within each of the three families), $\omega:=\exp (\pi i / m)$ is an $m$ th root of -1 , and $(r: s)$ is a point in $\mathbb{P}^{1}$ (cf. [5]). Thus, the components of $V[\alpha]$ are the images of the $3 \mathrm{~m}^{2}$ straight lines contained in the covering Fermat surface $\Phi[m]$, the components of the ramification locus of the covering $\Phi[\alpha] \rightarrow \Phi$, and the exceptional divisors arising from the resolution of singularities.

\section{The topology of a Delsarte surface}

In this section, we discuss a few simple topological properties of the Delsarte surface $\Phi[\alpha]$ and divisor $V[\alpha]$ defined by a finite quotient $\alpha: \mathbb{G} \rightarrow G$. In particular, we reduce most statements to the study of certain modules $\mathrm{A}[\alpha]$ or $\mathrm{B}[\alpha]$.

\subsection{The fundamental group: proof of Theorem 1.4}

The expression for the group $\pi_{1}(\Phi[\alpha])$ in terms of $\alpha$ is found in [2], and the statement that $\pi_{1}(\Phi[\alpha])=0$ for Fermat surfaces and unramified or diagonal Delsarte surfaces is immediate. We postpone the case of cyclic Delsarte surfaces until Section 5.2, where the necessary framework is introduced.

In general, we can assume that the kernel $\operatorname{Ker} \alpha$ is generated by three vectors $v_{i}:=t_{1}^{m_{i 1}} t_{2}^{m_{i 2}} t_{3}^{m_{i 3}}, i=1,2,3$, so that the matrix $\left[m_{i j}\right]$ is upper triangular,

$$
\left[m_{i j}\right]=\left[\begin{array}{ccc}
m_{11} & m_{12} & m_{13} \\
0 & m_{22} & m_{23} \\
0 & 0 & m_{33}
\end{array}\right] .
$$

Then $\mathbb{G}_{23} \cap \operatorname{Ker} \alpha$ contains $v_{3}$ and $v_{2}$, and $\mathbb{G}_{13} \cap \operatorname{Ker} \alpha$ contains $v_{3}$ and a product of the form $v_{1}^{r} v_{2}^{s}, r \neq 0$. Hence, $\pi_{1}(\Phi[\alpha])$ is a cyclic group (generated by $t_{1}$ ) of order at most $r$. On the other hand, from the expression in the statement, it is clear that $\pi_{1}(\Phi[\alpha])$ is a subquotient of the group $n \mathbb{G} / m \mathbb{G}$ of exponent ht $\alpha$, where $m:=\exp G$ and $n$ is as in the definition of ht $\alpha$ (see Section 1.2).

\subsection{The reduction}

Our proof of Theorems 1.5 and 1.7 is based on the following homological reduction of the problem.

\section{THEOREM 3.1}

Let $D$ be a divisor in a smooth compact analytic surface $X$, and let $K(X, D):=$ $\operatorname{Ker}\left[\kappa_{*}: H_{1}(X \backslash D) \rightarrow H_{1}(X)\right]$ be the kernel of the homomorphism $\kappa_{*}$ induced by 
the inclusion. Then there are canonical isomorphisms

$$
\operatorname{Tors} K(X, D)=\operatorname{Hom}(\mathbf{T}\langle D\rangle, \mathbb{Q} / \mathbb{Z}), \quad K(X, D) / \operatorname{Tors}=\operatorname{Hom}(\mathbf{K}\langle D\rangle, \mathbb{Z}) .
$$

Proof

The inclusion homomorphism $\kappa_{*}: H_{1}(X \backslash D) \rightarrow H_{1}(X)$ is Poincaré dual to the homomorphism $\beta$ in the following exact sequence of pair $(X, D)$ :

$$
\longrightarrow H^{2}(X) \stackrel{\iota^{*}}{\longrightarrow} H^{2}(D) \longrightarrow H^{3}(X, D) \stackrel{\beta}{\longrightarrow} H^{3}(X) \longrightarrow .
$$

Hence, $K(X, D)=$ Coker $\iota^{*}$, and both statements are immediate (cf. [2]), using the definition of the Ext groups in terms of projective resolutions and the canonical isomorphism $\operatorname{Ext}(A, \mathbb{Z})=\operatorname{Hom}(A, \mathbb{Q} / \mathbb{Z})$ for any finite abelian group $A$.

\subsection{The modules $\mathrm{A}[\alpha]$ and $\mathrm{B}[\alpha]$}

The groups $H_{1}\left(\Phi^{\circ}[\alpha]\right)=H_{1}(\Phi[\alpha] \backslash V[\alpha])$ for Delsarte surfaces were computed in [2], using the covering $\Phi^{\circ}[\alpha] \rightarrow \Phi^{\circ}$ and the presentation of the fundamental group $\pi_{1}\left(\Phi^{\circ}\right)$ given by the Zariski-van Kampen theorem. Let

$$
\Lambda:=\mathbb{Z}[\mathbb{G}]=\mathbb{Z}\left[t_{1}^{ \pm 1}, t_{2}^{ \pm 1}, t_{3}^{ \pm 1}\right]=\mathbb{Z}\left[t_{0}, t_{1}, t_{2}, t_{3}\right] /\left(t_{0} t_{1} t_{2} t_{3}-1\right)
$$

be the ring of Laurent polynomials. The deck translation action of the covering makes $H_{1}\left(\Phi^{\circ}[0]\right)$ a $\Lambda$-module, which is computed by the complex $0 \rightarrow \mathrm{A}[0] \rightarrow$ $\Lambda \rightarrow 0$ of $\Lambda$-modules defined as follows: $\mathrm{A}[0]$ is the $\Lambda$-module generated by six elements $a_{i}, c_{j}, i, j=1,2,3$, subject to the relations

$$
\begin{aligned}
\left(t_{2} t_{3}-1\right) c_{1}=\left(t_{1} t_{3}-1\right) c_{2}=\left(t_{1} t_{2}-1\right) c_{3} & =0, \\
\left(t_{3}-1\right) c_{1}+\left(t_{3}-1\right) a_{2}-\left(t_{2}-1\right) a_{3} & =0, \\
\left(t_{3}-1\right) c_{2}+\left(t_{3}-1\right) a_{1}-\left(t_{1}-1\right) a_{3} & =0, \\
\left(t_{1}-1\right) c_{3}+\left(t_{1}-1\right) a_{2}-\left(t_{2}-1\right) a_{1} & =0,
\end{aligned}
$$

and the boundary $\partial: \mathrm{A}[0] \rightarrow \Lambda$ is

$$
\partial a_{i}=\left(t_{i}-1\right), \quad \partial c_{j}=0, \quad i, j=1,2,3 .
$$

Here, $c_{i}$ is the cycle represented by any lift of a meridian about the line $L_{i} \subset \Phi$, $i=1,2,3$, whereas $a_{i}$ is merely a chain (not a cycle) projecting to a meridian about the component $R_{i} \subset \Phi$ of the ramification locus, $i=1,2,3$.

Now, given an epimorphism $\alpha: \mathbb{G} \rightarrow G$, let $\Lambda[\alpha]:=\mathbb{Z}[G]$, and consider the induced ring homomorphism $\Lambda \rightarrow \Lambda[\alpha]$. It makes $\Lambda[\alpha]$ a $\Lambda$-module, so that we can define $\mathrm{A}[\alpha]:=\mathrm{A}[0] \otimes_{\Lambda} \Lambda[\alpha]$. In other words, $\mathrm{A}[\alpha]$ is obtained from $\mathrm{A}[0]$ by adding to (3.2)-(3.5) the defining relations of $G$ in the basis $\left\{t_{1}, t_{2}, t_{3}\right\}$. Then, the computation in [2] can be summarized in the form of an exact sequence

$$
0 \longrightarrow H_{1}\left(\Phi^{\circ}[\alpha]\right) \longrightarrow \mathrm{A}[\alpha] \stackrel{\partial}{\longrightarrow} \Lambda[\alpha] \longrightarrow \mathbb{Z} \longrightarrow 0 .
$$

Since the group ring $\Lambda[\alpha]$ is free, it follows that Tors $H_{1}\left(\Phi^{\circ}[\alpha]\right)=\operatorname{Tors} \mathrm{A}[\alpha]$. 
Consider the divisor $D:=V[\alpha] \subset X:=\Phi[\alpha]$. The inclusion homomorphism $\kappa_{*}$ in Theorem 3.1 factors through the free abelian group

$$
H_{1}(\Phi[\alpha] \backslash R[\alpha])=\pi_{1}(\Phi[\alpha] \backslash R[\alpha])=\operatorname{Ker} \alpha \cong \mathbb{Z}^{3} ;
$$

hence, Tors Ker $\kappa_{*}=\operatorname{Tors} H_{1}\left(\Phi^{\circ}[\alpha]\right)$. Since $H_{1}(\Phi[\alpha])$ is finite (see Theorem 1.4), we have $\operatorname{rkKer} \kappa_{*}=\operatorname{rk} H_{1}\left(\Phi^{\circ}[\alpha]\right)$; that is, for Delsarte surfaces, in both statements in the conclusion of Theorem 3.1 the group $K(X, D)=\operatorname{Ker} \kappa_{*}$ can be replaced with $H_{1}\left(\Phi^{\circ}[\alpha]\right)$. Furthermore, repeating the argument in [2] (or merely patching the lines $L[\alpha]$, thus sending each generator $c_{i}$ to 0 ), one can easily see that the homology $H_{0}=\mathbb{Z}$ and $H_{1}=\operatorname{Ker} \alpha$ of the space $\Phi[\alpha] \backslash R[\alpha]$ are computed by the complex $0 \rightarrow \mathrm{A}[\alpha] / \mathrm{B}[\alpha] \rightarrow \Lambda[\alpha] \rightarrow 0$, where $\mathrm{B}[\alpha] \subset \mathrm{A}[\alpha]$ is the $\Lambda[\alpha]$-submodule generated by $c_{1}, c_{2}, c_{3}$. In other words, the inclusion $\Phi^{\circ}[\alpha] \hookrightarrow$ $\Phi[\alpha] \backslash R[\alpha]$ induces a map of (3.7) to the exact sequence

$$
0 \longrightarrow \operatorname{Ker} \alpha \longrightarrow \mathrm{A}[\alpha] / \mathrm{B}[\alpha] \stackrel{\partial}{\longrightarrow} \Lambda[\alpha] \longrightarrow \mathbb{Z} \longrightarrow 0 ;
$$

by the 5 -lemma, this map is an epimorphism. Comparing the two sequences and summarizing, we can restate Theorem 3.1 as

$$
\begin{aligned}
\operatorname{Hom}(\mathbf{T}[\alpha], \mathbb{Q} / \mathbb{Z}) & =\operatorname{Tors} H_{1}\left(\Phi^{\circ}[\alpha]\right)=\operatorname{Tors} \mathrm{A}[\alpha]=\operatorname{Tors} \mathrm{B}[\alpha], \\
\operatorname{rk} \mathbf{K}[\alpha] & =\operatorname{rkA}[\alpha]-|G|+1=\operatorname{rkB}[\alpha]+3 .
\end{aligned}
$$

\subsection{Generators of the torsion}

An explicit generating set for the primitive hull $\tilde{\mathbf{S}}[\alpha]:=(\mathbf{S}[\alpha] \otimes \mathbb{Q}) \cap \operatorname{NS}(\Phi[\alpha])$ can be described in terms of the discriminant form. We outline this description, in the hope that it may be useful in the future.

The lattice $\mathbf{S}[\alpha]$ has a vector of positive square (e.g., the hyperplane section class); hence, the Hodge index theorem implies that $\mathbf{S}[\alpha]$ is nondegenerate and its dual group $\mathbf{S}^{*}$ can be identified with a subgroup of $\mathbf{S}[\alpha] \otimes \mathbb{Q}$ :

$$
\mathbf{S}^{*}:=\operatorname{Hom}(\mathbf{S}[\alpha], \mathbb{Z})=\{x \in \mathbf{S}[\alpha] \otimes \mathbb{Q} \mid x \cdot y \in \mathbb{Z} \text { for all } y \in \mathbf{S}[\alpha]\} .
$$

This identification gives rise to an inclusion $\mathbf{S}[\alpha] \subset \mathbf{S}^{*}$ and to the discriminant group discr $\mathbf{S}[\alpha]:=\mathbf{S}^{*} / \mathbf{S}[\alpha]$ (see [4]). The latter is a finite abelian group equipped with a nondegenerate symmetric $\mathbb{Q} / \mathbb{Z}$-valued bilinear form, namely, the descent of the $\mathbb{Q}$-valued extension of the intersection index form from $\mathbf{S}[\alpha]$ to $\mathbf{S}^{*}$. Since $\tilde{\mathbf{S}}[\alpha]$ is also an integral lattice, there are natural inclusions

$$
\mathbf{S}[\alpha] \subset \tilde{\mathbf{S}}[\alpha] \subset \tilde{\mathbf{S}}^{*}:=\operatorname{Hom}(\tilde{\mathbf{S}}[\alpha], \mathbb{Z}) \subset \mathbf{S}^{*} ;
$$

hence, the extension $\tilde{\mathbf{S}}[\alpha] \supset \mathbf{S}[\alpha]$ is uniquely determined by either of the subgroups

$$
\mathcal{K}:=\tilde{\mathbf{S}}[\alpha] / \mathbf{S}[\alpha] \subset \mathcal{K}^{\perp}:=\tilde{\mathbf{S}}^{*} / \mathbf{S}[\alpha] \subset \operatorname{discr} \mathbf{S}[\alpha] .
$$

Indeed, the subgroups $\mathcal{K} \subset \mathcal{K}^{\perp}$ are the orthogonal complements of each other (in particular, $\mathcal{K}$ is isotropic), and

$$
\tilde{\mathbf{S}}[\alpha]=\{x \in \mathbf{S}[\alpha] \otimes \mathbb{Q} \mid x \bmod \mathbf{S}[\alpha] \in \mathcal{K}\} .
$$

For further details concerning discriminant forms and lattice extensions, see [4]. 
Consider the $\Lambda[\alpha]$-module $\tilde{\mathrm{B}}[\alpha]$ generated by $c_{1}, c_{2}, c_{3}$ subject to relations (3.2). The geometric description found in [2] establishes a canonical, up to the coordinate action of $\mathbb{G}$, homomorphism $\tilde{\mathrm{B}}[\alpha] \rightarrow H^{2}(V[\alpha])$ of $\Lambda[\alpha]$-modules, which restricts to an isomorphism $\tilde{\mathrm{B}}[\alpha]=H^{2}\left(L^{\prime}[\alpha]\right)$, where $L^{\prime}[\alpha]$ is the proper transform of $L$ in $\Phi[\alpha]$. If $\alpha=m \in \mathbb{N}_{+}$, then the reference point in $\Phi[m]$ can be chosen so that (see (2.3) for the notation)

$$
c_{1} \mapsto\left[L_{1}\left(1, \omega^{-2}\right)\right]^{*}, \quad c_{2} \mapsto\left[L_{2}\left(1, \omega^{-2}\right)\right]^{*}, \quad c_{3} \mapsto\left[L_{3}(1,1)\right]^{*} .
$$

In general, we use, in addition, the natural identifications $\tilde{\mathrm{B}}[\alpha]=\tilde{\mathrm{B}}[m] \otimes_{\Lambda} \Lambda[\alpha]$ and $H^{2}\left(L^{\prime}[\alpha]\right)=H^{2}(L[m]) \otimes_{\Lambda} \Lambda[\alpha]$.

Consider the modules

$$
\mathrm{K}^{\prime}:=\operatorname{Ker}[\tilde{\mathrm{B}}[\alpha] \rightarrow \mathrm{B}[\alpha]] \subset \mathrm{K}:=\operatorname{Ker}[\tilde{\mathrm{B}}[\alpha] \rightarrow \mathrm{B}[\alpha] / \text { Tors }] .
$$

It is immediate from the construction (with (3.8) taken into account) that the group $\mathrm{K} / \mathrm{K}^{\prime}$ is canonically isomorphic to $\mathbf{S}^{*} / \tilde{\mathbf{S}}^{*}$. The homomorphism $\mathrm{K} \rightarrow$ $\operatorname{discr} \mathbf{S}[\alpha]$ is easily computed using (3.10) and the intersection matrix of the components of $V[\alpha]$ (see, e.g., [5]), and the subgroup $\mathcal{K}^{\perp} \subset \operatorname{discr} \mathbf{S}[\alpha]$ defining the extension $\tilde{\mathbf{S}}[\alpha] \supset \mathbf{S}[\alpha]$ as described above is found as the image of $\mathrm{K}^{\prime}$.

\section{Proof of Theorem 1.5}

Throughout this section, we consider a finite quotient $\alpha: \mathbb{G} \rightarrow G$ and fix the notation $m:=\exp G$.

\subsection{Alternative proof of Theorem 1.7(1)}

This proof repeats almost literally the one found in [2], except that we analyze the module $\mathrm{B}[\alpha]$ instead of $\mathrm{A}[\alpha]$. This analysis (slightly more thorough than in [2]) is used in the sequel.

Assume that $\alpha=m: \mathbb{G} \rightarrow G=\mathbb{G} / m \mathbb{G}$, and consider the filtration

$$
0=\mathrm{B}_{0} \subset \mathrm{B}_{1} \subset \mathrm{B}_{2} \subset \mathrm{B}_{3} \subset \mathrm{B}_{4}:=\mathrm{B}[\alpha],
$$

where

- $\mathrm{B}_{3}$ is generated by $c_{1}^{\prime}:=\left(t_{3}-1\right) c_{1}, c_{2}^{\prime}:=\left(t_{3}-1\right) c_{2}, c_{3}^{\prime}:=\left(t_{1}-1\right) c_{3}$,

- $\mathrm{B}_{2}$ is generated by $c_{1}^{\prime \prime}:=\left(t_{1}-1\right) c_{1}^{\prime}, c_{2}^{\prime \prime}:=\left(t_{2}-1\right) c_{2}^{\prime}, c_{3}^{\prime \prime}:=\left(t_{3}-1\right) c_{3}^{\prime}$, and

- $\mathrm{B}_{1}$ is generated by the element $u:=\left(t_{2}-t_{3}^{-1}\right) c_{2}^{\prime \prime}$.

It is immediate that (see (3.2))

$$
\mathbb{Z}\left[G_{23}\right] c_{1} \oplus \mathbb{Z}\left[G_{13}\right] c_{2} \oplus \mathbb{Z}\left[G_{12}\right] c_{3}=\mathrm{B}_{4} / \mathrm{B}_{3} .
$$

The other relations do not affect this quotient. Furthermore, as obviously $\tilde{\varphi}_{m}\left(t_{3}\right) c_{1}^{\prime}=\tilde{\varphi}_{m}\left(t_{3}\right) c_{2}^{\prime}=\tilde{\varphi}_{m}\left(t_{1}\right) c_{3}^{\prime}=0$, we have an epimorphism

$$
\left(\mathbb{Z}\left[G_{01}\right] / \tilde{\varphi}_{m}\right) c_{1}^{\prime} \oplus\left(\mathbb{Z}\left[G_{02}\right] / \tilde{\varphi}_{m}\right) c_{2}^{\prime} \oplus\left(\mathbb{Z}\left[G_{03}\right] / \tilde{\varphi}_{m}\right) c_{3}^{\prime} \longrightarrow \mathrm{B}_{3} / \mathrm{B}_{2} .
$$

In $\mathrm{B}_{2}$, we have a relation

$$
c_{1}^{\prime \prime}=c_{2}^{\prime \prime}+c_{3}^{\prime \prime} ;
$$


it is the linear combination $\left(t_{1}-1\right)(3.3)-\left(t_{2}-1\right)(3.4)-\left(t_{3}-1\right)(3.5)$. Multiplying this by $\left(t_{2}-t_{3}^{-1}\right)$ and using (3.2), we have

$$
u:=\left(t_{2}-t_{3}^{-1}\right) c_{2}^{\prime \prime}=-\left(t_{2}-t_{3}^{-1}\right) c_{3}^{\prime \prime} .
$$

Hence, using (3.2) again, we obtain epimorphisms

$$
\begin{aligned}
\left(\mathbb{Z}\left[G_{3}\right] / \tilde{\varphi}_{m}\right) c_{2}^{\prime \prime} \oplus\left(\mathbb{Z}\left[G_{2}\right] / \tilde{\varphi}_{m}\right) c_{3}^{\prime \prime} \longrightarrow \mathrm{B}_{2} / \mathrm{B}_{1}, \\
\left(\mathbb{Z}\left[G_{1}\right] / \tilde{\varphi}_{m}\right) u \longrightarrow \mathrm{B}_{1} \quad(\text { for } m \text { odd }) .
\end{aligned}
$$

If $m=2 k$ is even, then arguing as in [2] we can refine (4.4) to

$$
\left(\mathbb{Z}\left[G_{1}\right] / \tilde{\varphi}_{k}\left(t^{2}\right)\right) u \longrightarrow \mathrm{B}_{1} \quad(\text { for } m=2 k \text { even }),
$$

where $t:=t_{0}=t_{1}=t_{2}^{-1}=t_{3}^{-1}$. Indeed, since $t_{2} u=t_{3} u=t_{1}^{-1} u$, by induction for $r \in \mathbb{Z}$ we have

$$
t_{2}^{r} c_{2}^{\prime \prime}=t_{1}^{r} c_{2}^{\prime \prime}+t_{2}^{1-r} \tilde{\varphi}_{r}\left(t_{2}^{2}\right) u .
$$

Summing up and using the fact that $\tilde{\varphi}_{m}\left(t_{1}\right) c_{2}^{\prime \prime}=\tilde{\varphi}_{m}\left(t_{2}\right) c_{2}^{\prime \prime}=0$ and the identity

$$
t^{m-2} \sum_{r=0}^{m-1} t^{1-r} \tilde{\varphi}_{r}\left(t^{2}\right)=t \tilde{\varphi}_{k-1}\left(t^{2}\right) \tilde{\varphi}_{m}(t)+\tilde{\varphi}_{k}\left(t^{2}\right), \quad m=2 k,
$$

which is easily established by multiplying both sides by $t^{2}-1$, we immediately conclude that $\tilde{\varphi}_{k}\left(t_{2}^{2}\right) u=0$.

Since $\alpha=m \in \mathbb{N}_{+}$, we have isomorphisms $G_{i j} \cong G_{i} \cong \mathbb{Z} / m$, and hence, all rings $\mathbb{Z}\left[G_{*}\right] / \tilde{\varphi}_{m}$ in (4.3) and (4.4) are free abelian groups of rank $m-1$. If $m=2 k$ is even, then the ring $\mathbb{Z}\left[G_{1}\right] / \tilde{\varphi}_{k}\left(t^{2}\right)$ in (4.5) is a free abelian group of rank $m-2$. Thus, summing up, we have $\ell(\mathrm{B}[\alpha]) \leq 9 m-6-\delta[\alpha]$. On the other hand, due to (3.9) and $[7], \operatorname{rkB}[\alpha]=9 m-6-\delta[\alpha]$. Hence, Tors $\mathrm{B}[\alpha]=0$.

\section{COROLLARY 4.6 (OF THE PROOF)}

The $\Lambda[m]$-module $\mathrm{B}[m]$ can be defined by relations (3.2) and $c_{1}^{\prime \prime}=c_{2}^{\prime \prime}+c_{3}^{\prime \prime}$, where the $c_{i}^{\prime \prime}$ 's are the elements introduced in Section 4.1. Furthermore, all epimorphisms in (4.1)-(4.5) are isomorphisms.

\section{REMARK 4.7}

Corollary 4.6 does not extend to other finite quotients (cf. Section 6.4).

\subsection{Proof of Theorem 1.5}

In view of (3.9), the rank $\operatorname{rk} \mathbf{K}[\alpha]$ can be computed as $\operatorname{dim}_{\mathbb{C}}(\mathrm{B}[\alpha] \otimes \mathbb{C})+3$. The group algebra $\mathbb{C}[\mathbb{G} / m \mathbb{G}]$ is semisimple, and we have (see Section 4.1)

$$
\mathrm{B}[m] \otimes \mathbb{C}=\mathrm{B}_{1} \otimes \mathbb{C} \oplus\left(\mathrm{B}_{2} / \mathrm{B}_{1}\right) \otimes \mathbb{C} \oplus\left(\mathrm{B}_{3} / \mathrm{B}_{2}\right) \otimes \mathbb{C} \oplus\left(\mathrm{B}_{4} / \mathrm{B}_{3}\right) \otimes \mathbb{C} .
$$

The rank formula in the theorem is obtained by tensoring this expression by $\mathbb{C}[G]$ and using isomorphisms (4.1)-(4.5). 
Let $(i, j, k)$ be a permutation of $(1,2,3)$, and introduce the following parameters measuring the "inhomogeneity" of $\operatorname{Ker} \alpha$ :

- $m_{i}$ is the order of the image $\alpha\left(t_{i}\right)$ in $G$;

- $n_{i}$ is the order of the image of $t_{i}$ (or $\left.t_{0}\right)$ in $G / \alpha\left(t_{0} t_{i}\right)=G / \alpha\left(t_{j} t_{k}\right)$;

- $n_{j k}$ is the order of the image of $t_{j}$ (or $t_{k}$ ) in $G / \alpha\left(t_{0} t_{i}\right)=G / \alpha\left(t_{j} t_{k}\right)$;

- $\bar{n}_{i}:=n_{i} /\left|G_{j k}\right|=n_{j k} /\left|G_{0 i}\right|$;

- $p_{i}:=\operatorname{gcd}\left(n_{i}, n_{j k}\right)$ and $\bar{p}_{i}:=p_{i} /\left|G_{l}\right|, i=2,3, i+l=5$;

- $\bar{q}:=\operatorname{gcd}\left(p_{2}, p_{3}\right) /\left|G_{1}\right|$.

It is not difficult to see that all $\bar{n}_{i}, \bar{p}_{i}$, and $\bar{q}$ are integers. If $\delta[\alpha]=1$, then also introduce

- $\bar{s}:=s /\left|G_{1}\right|$, where $s:=\operatorname{gcd}\left(s_{2}, s_{3}\right)$ and $s_{i}:=\operatorname{lcm}\left(n_{i}, m_{i}\right), i=2,3$.

Note that $\bar{s}$ is an integer and $\bar{q} \mid \bar{s}$. If $\delta[\alpha]=0$, then we merely let $\bar{s}:=1$.

\section{LEMMA 4.8}

There is a filtration $0=T_{0} \subset T_{1} \subset T_{2} \subset T_{3}:=$ Tors $\mathrm{B}[\alpha]$ such that the quotient groups $T_{i} / T_{i-1}, i=1,2,3$, are subquotients of

$$
(\mathbb{Z} / \bar{q}) \oplus(\mathbb{Z} / \bar{s}), \quad\left(\mathbb{Z} / \bar{p}_{2}\right) \oplus\left(\mathbb{Z} / \bar{p}_{3}\right), \quad\left(\mathbb{Z} / \bar{n}_{1}\right) \oplus\left(\mathbb{Z} / \bar{n}_{2}\right) \oplus\left(\mathbb{Z} / \bar{n}_{3}\right),
$$

respectively. In particular, $\ell(\operatorname{Tors} \mathrm{B}[\alpha]) \leq 6+\delta[\alpha]$.

Proof

Over $\Lambda[m]$, the tensor product does not need to be exact, but we still have an epimorphism $\mathrm{B}[m] \otimes_{\Lambda[m]} \Lambda[\alpha] \rightarrow \mathrm{B}[\alpha]$, which induces an epimorphism of the torsion groups (as the ranks of the two modules, regarded as abelian groups, are equal). Using the same filtration as in Section 4.1, we obtain epimorphisms (4.1)(4.5), which also induce epimorphisms of the torsion subgroups. Then, define the member $T_{i} \subset$ Tors $\mathrm{B}[\alpha]$ of the filtration as the image of $\mathrm{B}_{i}, i=0,1,2,3$.

The group rings $\mathbb{Z}\left[G_{*}\right]$ in (4.1) are torsion-free; hence, indeed, $T_{3}=$ Tors $\mathrm{B}[\alpha]$. Let $(i, j, k)$ be a permutation of $(1,2,3)$. In (4.2), each generator $c_{i}^{\prime}$ is annihilated by $\tilde{\varphi}_{n_{j k}}\left(t_{j}\right)$, and we can refine the corresponding summand to $\left(\mathbb{Z}\left[G_{0 i}\right] / \tilde{\varphi}_{n_{j k}}\right) c_{i}^{\prime}$. Let $r_{i}:=\left|G_{0 i}\right|$ be the order of the cyclic group $G_{0 i}$. Then $\tilde{\varphi}_{n_{j k}}=\bar{n}_{i} \tilde{\varphi}_{r_{i}}$ in $\mathbb{Z}\left[G_{0 i}\right]$, and $\mathbb{Z}\left[G_{0 i}\right] / \tilde{\varphi}_{r_{i}}$ is a free abelian group of rank $r_{i}-1$. Hence, Tors $\left(\mathbb{Z}\left[G_{0 i}\right] / \tilde{\varphi}_{n_{j k}}\right) c_{i}^{\prime}$ is a cyclic group $\mathbb{Z} / \bar{n}_{i} ;$ more precisely,

$$
\operatorname{ord}\left(\left(t_{k}^{r_{i}}-1\right) c_{i}\right) \text { in } \mathrm{B}[\alpha] / \mathrm{B}_{2} \text { divides } \bar{n}_{i}, \text { where } r_{i}:=\left|G_{0 i}\right| \text {. }
$$

Tensoring this element by $\mathbb{C}$, one can see that it does have finite order in $\mathrm{B}[\alpha] / \mathrm{B}_{2}$ but, in general, not in $\mathrm{B}[\alpha]$.

A similar argument applies to (4.3) and (4.4). In (4.3), the summand generated by $c_{i}^{\prime \prime}$ is refined to $\left(\mathbb{Z}\left[G_{l}\right] / \tilde{\varphi}_{p_{i}}\right) c_{i}^{\prime \prime}, l:=5-i$, the torsion of which is $\mathbb{Z} / \bar{p}_{i}$ :

$\operatorname{ord}\left(\left(t_{i}-1\right)\left(t_{l}^{r_{l}}-1\right) c_{i}\right)$ in $\mathrm{B}[\alpha] / \mathrm{B}_{1}$ divides $\bar{p}_{i}, i=2,3$, where $r_{l}:=\left|G_{l}\right|$. 
In (4.4), the module refines to $\left(\mathbb{Z}\left[G_{1}\right] / \tilde{\varphi}_{q}\right) u$, and we have $\operatorname{ord}\left(\left(t_{2}-t_{1}\right)\left(t_{2}^{r}-1\right)\left(t_{3}-1\right) c_{2}\right)$ in $\mathrm{B}[\alpha]$ divides $\bar{q}$, where $r:=\left|G_{1}\right|$.

If $\delta[\alpha]=1$ (equivalently, if both $m=2 k$ and $\left|G_{1}\right|=2 l$ are even), then we use (4.5) instead of (4.4). In addition to $\tilde{\varphi}_{q}(t) u=0$, we also have $\tilde{\varphi}_{s / 2}\left(t^{2}\right) u=0$ (cf. the end of Section 4.1). Since $\tilde{\varphi}_{s / 2}\left(t^{2}\right)=\bar{s} \tilde{\varphi}_{l}\left(t^{2}\right)$ and $\tilde{\varphi}_{q}(t)=\bar{q}(t+1) \tilde{\varphi}_{l}\left(t^{2}\right)$ in $\mathbb{Z}\left[G_{1}\right]$, we obtain an extra torsion term:

$$
\operatorname{ord}\left(\left(t_{2}-t_{1}\right) \tilde{\varphi}_{r}\left(-t_{2}\right)\left(t_{3}-1\right) c_{2}\right) \text { in } \mathrm{B}[\alpha] \text { divides } \bar{s} \text {, where } r:=\left|G_{1}\right| \text {. }
$$

Comparing the ranks, we conclude that the elements indicated above exhaust all torsion that may be present in $\mathrm{B}[\alpha]$.

\section{REMARK 4.9}

Note that Lemma 4.8 is merely an estimate on the size of $\mathbf{T}[\alpha]$. In particular, its conclusion depends on the order of the indices, and one may get a better estimate by permuting the indices $(0,1,2,3)$ (cf. Remark 6.1 and Section 6.4).

Denote by $\sigma: \Phi^{\prime}[m] \rightarrow \Phi[m]$ the Fermat surface $\Phi[m]$ blown up so that the projection $\pi: \Phi^{\prime}[m] \rightarrow \Phi[\alpha]$ is regular, and let $V^{\prime}[m]:=\sigma^{*} V[m]$.

\section{LEMMA 4.10}

The maps

$$
\mathrm{NS}(\Phi[\alpha]) \stackrel{\pi^{*}}{\longrightarrow} \mathrm{NS}\left(\Phi^{\prime}[m]\right) \stackrel{\pi_{*}}{\longrightarrow} \mathrm{NS}(\Phi[\alpha])
$$

respect the subgroups $\mathbf{S}[\alpha] \subset \mathrm{NS}(\Phi[\alpha])$ and $\mathbf{S}\left\langle V^{\prime}[m]\right\rangle \subset \mathrm{NS}\left(\Phi^{\prime}[m]\right)$. The composite map $\pi_{*} \circ \pi^{*}: \operatorname{NS}(\Phi[\alpha]) \rightarrow \mathrm{NS}(\Phi[\alpha])$ is the multiplication by $d:=m^{3} /|G|$.

\section{Proof}

The first statement is immediate from the definition of the divisors involved: set-theoretically, one has $V[\alpha]=\pi\left(V^{\prime}[m]\right)$ and $V^{\prime}[m]=\pi^{-1}(V[\alpha])$. The second statement is well known: since $\pi$ is a generically finite-to-one map of degree $d$, the assertion is geometrically obvious for the class of an irreducible curve $C \subset \Phi[\alpha]$ not contained in the ramification locus; then, it remains to observe that $\operatorname{NS}(\Phi[\alpha])$ is generated by such classes (e.g., very ample divisors).

By Lemma 4.10, we have induced maps

$$
\mathrm{NS}(\Phi[\alpha]) / \mathbf{S}[\alpha] \stackrel{\pi^{*}}{\longrightarrow} \mathrm{NS}\left(\Phi^{\prime}[m]\right) / \mathbf{S}\left\langle V^{\prime}[m]\right\rangle \stackrel{\pi_{*}}{\longrightarrow} \mathrm{NS}(\Phi[\alpha]) / \mathbf{S}[\alpha]
$$

whose composition $\pi_{*} \circ \pi^{*}$ is the multiplication by $d$. Since the group in the middle is torsion-free (see Theorem 1.7(1) and (2.1)), the group $\mathbf{T}[\alpha] \subset \operatorname{Ker} \pi^{*}$ is annihilated by $d$. Together with the estimate on $\ell(\mathbf{T}[\alpha])$ given by Lemma 4.8, this completes the proof of Theorem 1.5. 


\subsection{Proof of Theorem 1.7(4)}

The statement follows from Lemma 4.8, as one obviously has $\bar{n}_{i}=\bar{p}_{i}=\bar{q}=1$, $i=1,2,3$, and

$$
\bar{s}=\operatorname{lcm}_{1 \leq i<j \leq 3}\left(\operatorname{gcd}\left(m_{i}, m_{j}\right)\right) / \operatorname{gcd}\left(m_{1}, m_{2}, m_{3}\right) .
$$

In fact, using Corollary 4.6, one can easily show that $\operatorname{Tors}\left(\mathrm{B}[m] \otimes_{\Lambda} \Lambda[\alpha]\right)=\mathbb{Z} / \bar{s}$. Furthermore, numeric examples suggest that $\mathrm{B}[m] \otimes_{\Lambda} \Lambda[\alpha]=\mathrm{B}[\alpha]$ in the diagonal case (see Section 6.3). However, we do not know a proof of the latter statement.

\subsection{Proof of Addendum 1.6}

Obviously, $\mathbf{K}[\alpha] \subset$ ker $H_{2}(V[\alpha])$. On the other hand, the image $\mathbf{S}[\alpha]$ is a nondegenerate lattice (see the beginning of Section 3.4); hence, we also have the opposite inclusion $\mathbf{K}[\alpha] \supset$ ker $H_{2}(V[\alpha])$.

\subsection{Proof of Corollary 1.8}

According to [7], for any integer $m \in \mathbb{N}_{+}$prime to 6 , one has $\operatorname{NS}(\Phi[m]) \otimes \mathbb{Q}=$ $\mathbf{S}[m] \otimes \mathbb{Q}$. Then, by Lemma 4.10, a similar identity $\mathrm{NS}(\Phi[\alpha]) \otimes \mathbb{Q}=\mathbf{S}[\alpha] \otimes \mathbb{Q}$ holds for any finite quotient $\alpha: \mathbb{G} \rightarrow G$ with $|G|$ prime to 6 . It remains to observe that, for each surface $\Phi[\alpha]$ as in the statement,

- $\pi_{1}(\Phi[\alpha])=0$ (see Theorem 1.4); hence, Pic $\Phi[\alpha]=\operatorname{NS}(\Phi[\alpha])$, and

- $\mathbf{T}[\alpha]=0$ (see Theorem 1.7).

(If $\alpha$ is diagonal (cf. Theorem 1.7(4)), then the assumption that $|G|$ is prime to 6 implies also that $\delta[\alpha]=0$.) The last statement follows from Addendum 1.6.

\section{Cyclic Delsarte surfaces}

Throughout this section, we fix an epimorphism $\alpha: \mathbb{G} \rightarrow G$ and assume that $G$ is a finite cyclic group, $|G|=m$.

\subsection{The setup}

Fix a generator $t$ of $G$, and let $\alpha\left(t_{i}\right)=t^{m_{i}}, i=0,1,2,3$. Strictly speaking, $m_{0}, m_{1}$, $m_{2}, m_{3}$ are elements of $\mathbb{Z} / m$, but it is more convenient to regard them as nonnegative integers. Then $m_{0}+m_{1}+m_{2}+m_{3}=0 \bmod m$ and

$$
\operatorname{gcd}\left(m, m_{1}, m_{2}, m_{3}\right)=1 \text {. }
$$

For $i \neq j$, let $m_{i j}:=\operatorname{gcd}\left(m, m_{i}+m_{j}\right)$. We have $m_{i j}=m_{k l}$ whenever $(i, j, k, l)$ is a permutation of $(0,1,2,3)$, that is, there are three essentially distinct parameters $m_{i j}$.

It is easy to see that $\delta[\alpha]=1$ if and only if $m=0 \bmod 2$ and $m_{1} m_{2} m_{3}=$ $1 \bmod 2$. In view of (5.1),

$$
\operatorname{gcd}\left(m_{12}, m_{13}, m_{23}\right)=2^{\delta[\alpha]} .
$$

The following statement is an immediate consequence of (5.1) and (5.2). 


\section{LEMMA 5.3}

For a divisor $d \mid m, d>2$, the following two conditions:

(1) $d \mid m_{i}$ and $d \mid m_{j}$ for some $0 \leq i<j \leq 3$, or

(2) $d \mid m_{i j}$ and $d \mid m_{i k}$ for some permutation $(i, j, k)$ of $(1,2,3)$,

are mutually exclusive. Furthermore, $d$ may satisfy either (1) for at most one pair $i<j$ or (2) for at most one value of $i \in\{1,2,3\}$.

\subsection{Proof of Theorem 1.4 for cyclic Delsarte surfaces}

Due to the general expression for $\pi_{1}(\Phi[\alpha])$ given by Theorem 1.4, it suffices to show that, in the ring $\mathbb{Z} / m$, each solution to the equation $r_{1} m_{1}+r_{2} m_{2}+r_{3} m_{3}=0$ decomposes into a sum of solutions with at least one unknown $r_{i}=0$. Since $\mathbb{Z} / m=\bigoplus_{q} \mathbb{Z} / q$, the summation running over all maximal prime powers $q \mid m$, we can assume that $m$ itself is a prime power. Then, due to (5.1), at least one coefficient $m_{i}$ is prime to $m$. If, for example, $\operatorname{gcd}\left(m, m_{1}\right)=1$, that is, $m_{1}$ is invertible in $\mathbb{Z} / m$, then we obtain an equivalent equation $r_{1}=-r_{2} n_{2}-r_{3} n_{3}$, where $n_{i}:=m_{i} m_{1}^{-1}, i=1,2$, for which the decomposition statement is obvious.

\subsection{Invariant factors}

In the rest of this section, we prove Theorem 1.7(3) by analyzing the structure of the module $\mathrm{A}[\alpha]$ (see Remark 6.3 for an explanation). Introduce the notation

$$
\sigma:=t^{m}-1, \quad \sigma_{i}:=t^{m_{i}}-1, \quad \sigma_{i j}:=t^{m_{i j}}-1, \quad i, j=0,1,2,3, i \neq j .
$$

Recall that, for $p, q \in \mathbb{Z}$, one has $\operatorname{gcd}\left(t^{p}-1, t^{q}-1\right)=t^{\operatorname{gcd}(p, q)}-1$. Hence, the polynomials introduced are subject to the following divisibility relations:

$$
\begin{aligned}
& \left.\sigma_{i j} \mid \sigma \quad \text { for all } i \neq j \text { (by the definition of } m_{i j}\right), \\
& \operatorname{gcd}\left(\sigma, \sigma_{1}, \sigma_{2}, \sigma_{3}\right)=o:=t-1 \quad(\text { see }(5.1)), \\
& \operatorname{gcd}\left(\sigma_{i}, \sigma_{j}, \sigma_{i k}\right)=\operatorname{gcd}\left(\sigma_{i}, \sigma_{i j}, \sigma_{i k}\right)=o \quad \text { for }\{i, j, k\}=\{1,2,3\}, \\
& \operatorname{gcd}\left(\sigma_{12}, \sigma_{13}, \sigma_{23}\right)=\rho o, \quad \rho:=(t+1)^{\delta[\alpha]}(\operatorname{see}(5.2)) .
\end{aligned}
$$

(The third relation follows from the similar relations for the exponents $m_{*}$, which, in turn, are consequences of (5.1).) The gcd-type relations in (5.4) hold in the following strong ideal sense: each relation $\operatorname{gcd}\left(\beta_{1}, \beta_{2}, \ldots\right)=\beta$ means that the ideal $\mathrm{R} \beta_{1}+\mathrm{R} \beta_{2}+\cdots$ in the polynomial ring $\mathrm{R}:=\mathbb{Z}\left[t^{ \pm 1}\right]$ equals $\mathrm{R} \beta$. In other words, $\beta$ divides each $\beta_{i}$ in $\mathrm{R}$ and $\beta=\gamma_{1} \beta_{1}+\gamma_{2} \beta_{2}+\cdots$ for some polynomials $\gamma_{i} \in \mathrm{R}$. Hence, we have the same relations in $\mathbb{k} \mathrm{R}:=\mathbb{Z}\left[t^{ \pm 1}\right] \otimes \mathbb{k}=\mathbb{k}\left[t^{ \pm 1}\right]$, where $\mathbb{k}$ is a field of any characteristic.

We regard $\mathrm{A}[\alpha]$ as an $\mathrm{R}$-module. It is generated by $a_{1}, a_{2}, a_{3}, c_{1}, c_{2}, c_{3}$, and the defining relations are (3.3)-(3.5) with $t_{i}=t^{m_{i}}, i=1,2,3$, and

$$
\sigma a_{1}=\sigma a_{2}=\sigma a_{3}=\sigma_{23} c_{1}=\sigma_{13} c_{2}=\sigma_{12} c_{3}=0 .
$$

(The first three relations make $\mathrm{A}[\alpha]$ a $\mathbb{Z}[G]$-module, and the last three are (3.2) combined with $\sigma c_{i}=0, i=1,2,3$.) The relations in $\mathrm{A}[\alpha]$ are represented by the matrix 


$$
M:=\left[\begin{array}{cccccc}
0 & \sigma_{3} & -\sigma_{2} & \sigma_{3} & 0 & 0 \\
\sigma_{3} & 0 & -\sigma_{1} & 0 & \sigma_{3} & 0 \\
-\sigma_{2} & \sigma_{1} & 0 & 0 & 0 & \sigma_{1} \\
\sigma & 0 & 0 & 0 & 0 & 0 \\
0 & \sigma & 0 & 0 & 0 & 0 \\
0 & 0 & \sigma & 0 & 0 & 0 \\
0 & 0 & 0 & \sigma_{23} & 0 & 0 \\
0 & 0 & 0 & 0 & \sigma_{13} & 0 \\
0 & 0 & 0 & 0 & 0 & \sigma_{12}
\end{array}\right]
$$

Given a field $\mathbb{k}$, the reduction $\mathrm{A}[\alpha] \otimes \mathbb{k}$ is a finitely generated module over the principal ideal domain $\mathbb{k} R$; hence, it decomposes into a direct sum of cyclic modules,

$$
\mathrm{A}[\alpha] \otimes \mathbb{k} \cong \mathbb{k} \mathrm{R} / f_{1} \oplus \cdots \oplus \mathbb{k} \mathrm{R} / f_{6},
$$

where $f_{1}, \ldots, f_{6}$ are the invariant factors of $M \otimes \mathbb{k}$, that is, the diagonal elements of the Smith normal form of the matrix. Recall that $f_{1}\left|f_{2}\right| f_{3}\left|f_{4}\right| f_{5} \mid f_{6}$ are elements of $\mathbb{k R}$ that can be found as $f_{r}=\left(\operatorname{gcd} S_{r}\right) /\left(\operatorname{gcd} S_{r-1}\right), r=1, \ldots, 6$, where $S_{r}$ is the set of all $(r \times r)$-minors of $M \otimes \mathbb{k}$.

All nontrivial minors of $M$ are products of polynomials of the form $\left(t^{s}-1\right)$. Computing all $(r \times r)$-minors, $r=1, \ldots, 6$, we obtain six lengthy sequences $S_{r}$. Since we are interested in the greatest common divisors only, we use (5.4) (in the ideal sense as explained above) and simplify these sequences as described below.

Whenever a sequence $S$ contains a subsequence of the form

- $\beta \sigma, \beta \sigma_{1}, \beta \sigma_{2}, \beta \sigma_{3}$, or

- $\beta \sigma_{i}, \beta \sigma_{j}, \beta \sigma_{i k}$ for some $\{i, j, k\}=\{1,2,3\}$, or

- $\beta \sigma_{i}, \beta \sigma_{i j}, \beta \sigma_{i k}$ for some $\{i, j, k\}=\{1,2,3\}$,

where $\beta$ is a common factor, one can append to $S$ the product $\beta$. After all such additions have been made, one can shorten $S$ by removing all nontrivial multiples of any element $\beta^{\prime} \in S$. We repeat these two steps until $S$ stabilizes and then apply a similar procedure, replacing each subsequence $\beta \sigma_{12}, \beta \sigma_{13}$, $\beta \sigma_{23}$ with the product $\beta \rho o$. Denoting by $S_{r}^{\prime}$ the result of the simplification, we have

$$
\begin{aligned}
& S_{1}^{\prime}=\{o\}, \quad S_{2}^{\prime}=\left\{o^{2}\right\}, \quad S_{3}^{\prime}=\left\{o^{3}\right\}, \quad S_{4}^{\prime}=\left\{\rho o^{4}\right\}, \\
& S_{5}^{\prime}=\left\{\sigma \rho o^{4}, \sigma_{12} \sigma_{13} \sigma_{23} o^{2}, \sigma_{2} \sigma_{3} \sigma_{12} \sigma_{23} o, \sigma_{1} \sigma_{3} \sigma_{12} \sigma_{13} o, \sigma_{1} \sigma_{3} \sigma_{13} \sigma_{23} o\right\} .
\end{aligned}
$$

Another observation is the fact that $S_{6}$ is a subset of $\left\{\sigma \beta \mid \beta \in S_{5}\right\}$; hence, one has $\sigma\left(\operatorname{gcd} S_{5}\right) \mid \operatorname{gcd} S_{6}$. On the other hand, $\mathrm{A}[\alpha]$ is a $\mathbb{Z}[G]$-module and all its invariant factors are divisors of $\sigma$. Taking into account (5.6), we easily obtain all invariant factors (in any characteristic) except $f_{5}$ :

$$
f_{1}=f_{2}=f_{3}=o, \quad f_{4}=\rho o, \quad f_{6}=\sigma .
$$


5.4. The factor $f_{5}$ : the case $\mathbb{k}=\mathbb{Q}$

Let $\bar{\sigma}_{*}:=\sigma_{*} / o$, and cancel the common factor $o^{5}$, converting $S_{5}^{\prime}$ to the union

$$
S_{5}^{\prime \prime}:=\{\bar{\sigma} \rho\} \cup\left\{\bar{\sigma}_{12} \bar{\sigma}_{13} \bar{\sigma}_{23}, \bar{\sigma}_{2} \bar{\sigma}_{3} \bar{\sigma}_{12} \bar{\sigma}_{23}, \bar{\sigma}_{1} \bar{\sigma}_{3} \bar{\sigma}_{12} \bar{\sigma}_{13}, \bar{\sigma}_{1} \bar{\sigma}_{3} \bar{\sigma}_{13} \bar{\sigma}_{23}\right\} .
$$

Over $\mathbb{Q}$, the irreducible factors of $\sigma$ are distinct cyclotomic polynomials $\psi_{d}, d \mid m$, and a factor $\psi_{d}, d>2$ may appear in gcd $S_{5}^{\prime \prime}$ at most once. Since $\psi_{d} \mid \bar{\sigma}_{12} \bar{\sigma}_{13} \bar{\sigma}_{23}$, one has $d \mid m_{i j}$ for some $1 \leq i<j \leq 3$. It remains to consider the three possibilities case by case and analyze the remaining three elements of $S_{5}^{\prime \prime}$. Using the relations between $m_{*}$ (mainly, the fact that $\left.\operatorname{gcd}\left(m_{i}, m_{i j}\right)=\operatorname{gcd}\left(m, m_{i}, m_{j}\right)\right)$, we arrive at the following restrictions to $d$ :

(1) $d \mid m_{i}$ and $d \mid m_{j}$ for some $1 \leq i<j \leq 3$, or

(2) $d \mid m_{i j}$ and $d \mid m_{i k}$ for some permutation $(i, j, k)$ of $(1,2,3)$, or

(3) $d \mid m_{i}$ and $d \mid m_{j k}$ for some permutation $(i, j, k)$ of $(1,2,3)$.

The substitution $m_{j k} \mapsto m_{i 0}=-m_{j k} \bmod m$ converts (3) to (1) with $(i, j)=$ $(i, 0)$. Hence, $\operatorname{gcd} S_{5}^{\prime}=f_{5} \rho o^{4}$ with

$$
f_{5}=\prod \psi_{d}(t)
$$

where the product runs over all divisors $d \mid m$ satisfying conditions (1) or (2) in Lemma 5.3. (In the special case $d=2$ and $\delta[\alpha]=1$, the greatest common divisor contains two copies of $(t+1)$; one of them is $\rho$, and the other is counted in the product (5.8) for $f_{5}$. An extra factor $o=\psi_{1}(t)$ is also counted in the product.)

\section{REMARK 5.9}

According to (5.7) and (5.8), $\operatorname{rkA}[\alpha]=m+4+\delta[\alpha]+\sum_{d} \phi(d)$, where $\phi(d)=$ $\operatorname{deg} \psi_{d}$ is Euler's totient function and the summation runs over all divisors $d \mid m$ satisfying conditions (1) or (2) in Lemma 5.3. Since $n=\sum_{d \mid n} \phi(d)$ for $n \in \mathbb{N}_{+}$, this expression translates to $\operatorname{rk} \mathrm{A}[\alpha]=m-4-\delta[\alpha]+\sum_{i<j} d_{i j}+\sum_{i} d_{i}$ (using Lemma 5.3 again), where

- $d_{i j}:=\operatorname{gcd}\left(m, m_{i}, m_{j}\right)=\left|G_{i j}\right|$ for $0 \leq i<j \leq 3$, and

- $d_{i}:=\operatorname{gcd}\left(m_{i j}, m_{i k}\right)=\left|G_{i}\right|$ for $i=1,2,3$ and $\{i, j, k\}=\{1,2,3\}$.

This agrees with (3.9) and Theorem 1.5.

\subsection{The factor $f_{5}$ : the case $\mathbb{k}=\mathbb{F}_{p}$}

Fix a prime $p>0$, and compute $f_{5}$ over $\mathbb{F}_{p}$. This time, the cyclotomic polynomials $\psi_{d}$ may be reducible. However, for any pair $n, d \in \mathbb{N}_{+}$with $\operatorname{gcd}(d, p)=1$, one still has $\psi_{d} \mid\left(t^{n}-1\right)$ if $d \mid n$ and $\operatorname{gcd}\left(\psi_{d}, t^{n}-1\right)=1$ otherwise. Thus, if $p$ is prime to $m$ (and hence $\sigma_{m}$ is square-free), then the computation runs exactly as in Section 5.4 and we arrive at (5.8).

In general, let $m_{*}=m_{*}^{\prime} q_{*}$, where $q_{*}$ is a power of $p$ and $m_{*}^{\prime}$ is prime to $p$. Then, $\sigma_{*}=\left(\sigma_{*}^{\prime}\right)^{q_{*}}$, where $\sigma_{*}^{\prime}:=t^{m_{*}^{\prime}}-1$ is square-free. To reduce the number of cases and simplify the argument, note that the isomorphism class of the module $\mathrm{A}[\alpha] \otimes \mathbb{F}_{p}$ and, hence, its invariant factors depend on $m$ and the unordered quadruple $\left(m_{0}, m_{1}, m_{2}, m_{3}\right)$ only. Thus, permuting the indices, we can add to $S_{5}^{\prime}$ 
all products of the form $\sigma_{i} \sigma_{j} \sigma_{i j} \sigma_{i k} O$, where $(i, j, k)$ runs over all three-element arrangements of $\{0,1,2,3\}$. Denote this new set by $S_{5}^{\prime \prime}$.

Let $d^{\prime} \mid m^{\prime}, d^{\prime}>2$. Arguing as in Section 5.4, we conclude that $\psi_{d^{\prime}}$ divides $\operatorname{gcd} S_{5}^{\prime}$ if and only if

(1) $d^{\prime} \mid m_{i}^{\prime}$ and $d^{\prime} \mid m_{j}^{\prime}$ for some $0 \leq i<j \leq 3$, or

(2) $d^{\prime} \mid m_{i j}^{\prime}$ and $d^{\prime} \mid m_{i k}^{\prime}$ for some permutation $(i, j, k)$ of $(1,2,3)$.

As in Lemma 5.3, the two conditions are mutually exclusive and $d^{\prime}$ may satisfy either (1) for exactly one pair $i<j$ or (2) for exactly one value of $i$.

In case (1), assume that $(i, j)=(1,2)$ and $q_{1}=\min \left(q_{1}, q_{2}\right)$. Then $d^{\prime}$ divides $m_{1}^{\prime}, m_{2}^{\prime}, m_{12}^{\prime}$, and $m_{03}^{\prime}$, and $d^{\prime}$ does not divide any other of $m_{k}^{\prime}$ or $m_{k l}^{\prime}$. Considering the element $\sigma_{1} \sigma_{3} \sigma_{13} \sigma_{01} o \in S_{5}^{\prime \prime}$, we see that the multiplicity of $\psi_{d^{\prime}}$ in $\operatorname{gcd} S_{5}^{\prime}$ is at most (and hence equal to) $q^{\prime}:=\min \left(q, q_{1}\right)$, that is, the one given by (5.8) reduced modulo $p$. Indeed, for $\psi_{d^{\prime}}$, the product in (5.8) should be restricted to the divisors of $m$ of the form $d=d^{\prime} p^{r}$. By the assumption $q^{\prime}=\min \left(q, q_{1}, q_{2}\right)$, we have $1 \leq p^{r} \leq q^{\prime}$. Since

$$
\psi_{d^{\prime} p^{r}}=\left(\psi_{d^{\prime}}\right)^{p^{r}-p^{r-1}} \quad \text { for } r \geq 1,
$$

the exponents sum up to $q^{\prime}$.

In case (2), assume that $(i, j, k)=(1,2,3)$ and $q_{12} \leq q_{13}$. Then $d^{\prime}$ divides $m_{12}^{\prime}$, $m_{13}^{\prime}, m_{03}^{\prime}$, and $m_{02}^{\prime}$, and $d^{\prime}$ does not divide any other of $m_{l}^{\prime}$ or $m_{l n}^{\prime}$. Considering the element $\sigma_{1} \sigma_{2} \sigma_{12} \sigma_{01} o \in S_{5}^{\prime \prime}$, as in the previous case we conclude that the multiplicity of $\psi_{d^{\prime}}$ in $\operatorname{gcd} S_{5}^{\prime}$ is at most (and hence equal to) $q_{12}$, that is, the one given by (5.8).

If $d^{\prime}=1$, then the multiplicity of $\psi_{1}=o$ (in addition to the five copies present in each term automatically) is counted by a similar argument, using the fact that $d=p$ itself satisfies at most one of the two conditions in Lemma 5.3 and with at most one parameter set. The extra multiplicity is $\min \left(q, q_{i}, q_{j}\right)$ in case (1) or $\min \left(q_{i j}, q_{i k}\right)$ in case (2), that is, again the one given by (5.8) (where the product is to be restricted to the divisors $d \mid m$ that are powers of $p$ ). In the special case $p=2 \mid m$, assuming that $\delta[\alpha]=0$, the divisor $d=2$ is also covered by Lemma 5.3 and the previous argument applies.

As in Section 5.4, the case where $\delta[\alpha]=1$ and either $d^{\prime}=2$ or $p=2$ needs special attention, taking into account the common divisor 2 of all the $m_{i j}$ 's. For example, let $p=2$, and compute the multiplicity of $\rho=o$ in $\operatorname{gcd} S_{5}^{\prime}$. Since $\delta[\alpha]=1$, all the $m_{i}$ 's are odd, that is, $q_{i}=1$ for $i=0,1,2,3$ (see the beginning of Section 5.1). By (5.2), we can assume that $2=q_{12} \leq q_{13} \leq q_{23} \leq q$. Then it is immediate that the maximal power of $o$ dividing $\operatorname{gcd} S_{5}^{\prime}=\operatorname{gcd} S_{5}^{\prime \prime}$ is $o^{q_{13}+5}$ : this maximum is attained at the term $\sigma_{1} \sigma_{2} \sigma_{12} \sigma_{13} o$. Disregarding $o^{5}=\rho o^{4}$, we conclude that the multiplicity of $o$ in the invariant factor $f_{5} \in \mathbb{F}_{2} \mathrm{R}$ is $q_{13}$. On the other hand, it is easily seen that $q_{13}$ is the maximal power of 2 satisfying conditions (1) or (2) in Lemma 5.3; hence, $o^{q_{13}}=t^{q_{13}}-1$ is precisely the maximal power of $o$ dividing (5.8) mod 2. Further details are left to the reader.

Summarizing, we conclude that, for any prime $p$, the invariant factor $f_{5}$ of the $\mathbb{F}_{p} \mathrm{R}$-module $\mathrm{A}[\alpha] \otimes \mathbb{F}_{p}$ is merely the $(\bmod p)$-reduction of $(5.8)$. 


\subsection{End of the proof of Theorem 1.7(3)}

For each field $\mathbb{k}=\mathbb{Q}$ or $\mathbb{F}_{p}$,

$$
\operatorname{dim}(\mathrm{A}[\alpha] \otimes \mathbb{k})=\operatorname{deg} f_{1, \mathbb{k}}+\cdots+\operatorname{deg} f_{6, \mathbb{k}},
$$

where $f_{r, \mathbb{k}} \in \mathbb{k} \mathrm{R}, r=1, \ldots, 6$, are the invariant factors of $\mathrm{A}[\alpha] \otimes \mathbb{k}$. According to Sections 5.3-5.5, each $f_{r, \mathbb{k}}$ is the reduction to $\mathbb{k}$ of the monic polynomial $f_{r} \in \mathbb{Z}[t]$ given by (5.7) or (5.8). Hence, $\operatorname{dim}(\mathrm{A}[\alpha] \otimes \mathbb{k})$ does not depend on $\mathbb{k}$.

\section{Examples}

In conclusion, we mention a few numeric examples showing the sharpness of most estimates stated in Section 1.2. Most examples result from experiments with random matrices, and it appears that the presence of a nontrivial torsion in $\mathrm{B}[\alpha]$ is quite common. The input for the computation is a $(3 \times 3)$-matrix $M$ whose rows are the coordinates (in the basis $t_{1}, t_{2}, t_{3} \in \mathbb{G}$ ) of three vectors generating Ker $\alpha$. Usually, this matrix is in the form $\operatorname{diag}\left(m_{1}, m_{2}, m_{3}\right) M^{\prime}$, where diag is a diagonal matrix and $M^{\prime}$ is unimodular: in the experiments, the diagonal part was fixed while $M^{\prime}$ was chosen randomly.

To shorten the display, we represent the isomorphism class of the finite group $\mathbf{T}[\alpha]$ by the vector $T=\left[a_{i}\right]$ of its invariant factors, so that $\mathbf{T}[\alpha]=\bigoplus_{i} \mathbb{Z} / a_{i}$.

\subsection{Torsion groups of maximal length}

For the finite quotients $\alpha_{i}$ defined by the matrices $M_{i}:=D M_{i}^{\prime}$, where $D:=$ $\operatorname{diag}(1,8,8)$, one has

$$
\begin{aligned}
& M_{1}^{\prime}=\left[\begin{array}{lll}
4 & 7 & 1 \\
1 & 0 & 0 \\
0 & 1 & 0
\end{array}\right]: \quad \pi_{1}\left(\Phi\left[\alpha_{1}\right]\right)=\mathbb{Z} / 2, \quad T=[2,2,2,2,2,2,4], \\
& M_{2}^{\prime}=\left[\begin{array}{lll}
0 & 3 & 1 \\
1 & 0 & 0 \\
0 & 1 & 0
\end{array}\right]: \quad \pi_{1}\left(\Phi\left[\alpha_{2}\right]\right)=0, \quad T=[2,2,2,4] .
\end{aligned}
$$

If $D=\operatorname{diag}(1,8,16)$, then

$$
\begin{aligned}
& M_{3}^{\prime}=\left[\begin{array}{ccc}
4 & 1 & -1 \\
1 & 1 & 0 \\
1 & 0 & 0
\end{array}\right]: \quad \pi_{1}\left(\Phi\left[\alpha_{3}\right]\right)=\mathbb{Z} / 2, \quad T=[2,2,2,4,4,4,4], \\
& M_{4}^{\prime}=\left[\begin{array}{lll}
6 & 1 & 2 \\
1 & 0 & 1 \\
0 & 0 & 1
\end{array}\right]: \quad \pi_{1}\left(\Phi\left[\alpha_{4}\right]\right)=\mathbb{Z} / 4, \quad T=[2,4,4,4,4,8], \\
& M_{5}^{\prime}=\left[\begin{array}{lll}
1 & 0 & 3 \\
0 & 1 & 1 \\
0 & 0 & 1
\end{array}\right]: \quad \pi_{1}\left(\Phi\left[\alpha_{5}\right]\right)=0, \quad T=[4,4,4,4] .
\end{aligned}
$$


If $D=\operatorname{diag}(1,9,9)$ (and hence $\delta[\alpha]=0$ ), then

$$
\begin{aligned}
& M_{6}^{\prime}=\left[\begin{array}{ccc}
-3 & 1 & 2 \\
1 & 0 & 0 \\
0 & 0 & 1
\end{array}\right]: \quad \pi_{1}\left(\Phi\left[\alpha_{6}\right]\right)=\mathbb{Z} / 3, \quad T=[3,3,3,3,3,9], \\
& M_{7}^{\prime}=\left[\begin{array}{ccc}
-1 & 1 & 1 \\
0 & 1 & 1 \\
0 & 0 & 1
\end{array}\right]: \quad \pi_{1}\left(\Phi\left[\alpha_{7}\right]\right)=0, \quad T=[3,3,9] .
\end{aligned}
$$

Finally, for $D=\operatorname{diag}(2,9,9)$ one has

$$
\begin{aligned}
& M_{8}^{\prime}=\left[\begin{array}{ccc}
-4 & 2 & 1 \\
-3 & 1 & 0 \\
1 & 0 & 1
\end{array}\right]: \quad \pi_{1}\left(\Phi\left[\alpha_{8}\right]\right)=\mathbb{Z} / 3, \quad T=[3,3,3,3,3,3,9], \\
& M_{9}^{\prime}=\left[\begin{array}{ccc}
3 & 2 & 0 \\
1 & 1 & 0 \\
3 & 0 & -1
\end{array}\right]: \quad \pi_{1}\left(\Phi\left[\alpha_{9}\right]\right)=0, \quad T=[3,3,3,9] .
\end{aligned}
$$

\section{REMARK 6.1}

In most examples considered in this section, the estimate given by Lemma 4.8 does depend on the order of the indices (cf. Remark 4.9); often, even the best bound is larger than the actual size $|\mathbf{T}[\alpha]|$. In many cases, the epimorphism $\mathrm{B}[m] \otimes_{\Lambda} \Lambda[\alpha] \rightarrow \mathrm{B}[\alpha]$ is not an isomorphism (cf. Remark 4.7). Note also that, for the finite quotient $\alpha_{4}$, one has (cf. Lemma 4.8)

$$
T_{2} / T_{0} \cong T_{3} / T_{2} \cong \mathbb{Z} / 4 \oplus \mathbb{Z} / 4 \oplus \mathbb{Z} / 4,
$$

whereas $\exp \mathbf{T}\left[\alpha_{4}\right]=8$.

\subsection{The case of $|G|$ prime to 6}

In this case, one always has $\delta[\alpha]=0$. Let $\alpha_{i}$ be defined by a matrix $M_{i}:=D M_{i}^{\prime}$. If $D=\operatorname{diag}(1,5,25)$, then one has

$$
\begin{aligned}
& M_{1}^{\prime}=\left[\begin{array}{ccc}
2 & -1 & 6 \\
1 & 0 & 1 \\
0 & 0 & 1
\end{array}\right]: \quad \pi_{1}\left(\Phi\left[\alpha_{1}\right]\right)=\mathbb{Z} / 5, \quad T=[5,5,5,5,5,5], \\
& M_{2}^{\prime}=\left[\begin{array}{ccc}
2 & 0 & -1 \\
4 & 1 & -1 \\
1 & 0 & 0
\end{array}\right]: \quad \pi_{1}\left(\Phi\left[\alpha_{2}\right]\right)=0, \quad T=[5,5,5] .
\end{aligned}
$$

If $D=\operatorname{diag}(1,7,7)$, then

$$
\begin{aligned}
& M_{3}^{\prime}=\left[\begin{array}{lll}
1 & 2 & 5 \\
0 & 0 & 1 \\
1 & 1 & 0
\end{array}\right]: \quad \pi_{1}\left(\Phi\left[\alpha_{3}\right]\right)=\mathbb{Z} / 7, \quad T=[7,7,7,7,7,7], \\
& M_{4}^{\prime}=\left[\begin{array}{lll}
1 & 0 & 2 \\
1 & 0 & 1 \\
3 & 1 & 0
\end{array}\right]: \quad \pi_{1}\left(\Phi\left[\alpha_{4}\right]\right)=0, \quad T=[7,7,7] .
\end{aligned}
$$




\section{REMARK 6.2}

The examples in Sections 6.1 and 6.2 suggest that, under the additional assumption that $\pi_{1}(\Phi[\alpha])=0$, we have a better bound $\ell(\mathbf{T}[\alpha]) \leq 3+\delta[\alpha]$. It also appears that $\exp \mathbf{T}[\alpha]$ divides ht $\alpha$. We do not know a proof of these facts.

\subsection{Diagonal Delsarte surfaces}

We tested the diagonal finite quotients

$$
\alpha=(2,4,4),(2,6,6),(2,8,8),(4,6,12) .
$$

In all cases, the obvious epimorphism $\mathrm{B}[m] \otimes_{\Lambda} \Lambda[\alpha] \rightarrow \mathrm{B}[\alpha]$ is an isomorphism, that is, the torsion Tors $\mathrm{B}[\alpha]$ is the maximal allowed by Theorem 1.7(4) (see Section 4.3).

\subsection{Cyclic Delsarte surfaces}

The last example illustrates Remarks 4.7 and 4.9, showing that, in general, one may need to deal with the whole module $\mathrm{A}[\alpha]$ when computing the torsion. Let $\alpha: \mathbb{G} \rightarrow G$ be the finite quotient defined by the matrix

$$
M:=\left[\begin{array}{lll}
1 & 1 & 0 \\
3 & 0 & 3 \\
0 & 0 & 4
\end{array}\right] .
$$

It is immediate that $m=12$ and $G \cong \mathbb{Z} / m$ is a cyclic group; hence, Tors $\mathrm{B}[\alpha]=0$ (see (3.8) and Theorem 1.7(3)).

Let $\mathrm{B}^{\prime}[\alpha]:=\mathrm{B}[m] \otimes_{\Lambda} \Lambda[\alpha]$; by Corollary 4.6 , this $\Lambda[\alpha]$-module is defined by (3.2) and relation $c_{1}^{\prime \prime}=c_{2}^{\prime \prime}+c_{3}^{\prime \prime}$. Consider the filtrations $\mathrm{B}_{i} \subset \mathrm{B}[\alpha]$ and $\mathrm{B}_{i}^{\prime} \subset \mathrm{B}^{\prime}[\alpha]$, $i=0, \ldots, 4$, defined as in Section 4.1. Then, a straightforward computation shows that $\operatorname{Tors}\left(\mathrm{B}_{3} / \mathrm{B}_{2}\right)=\mathbb{Z} / 4 \oplus \mathbb{Z} / 2$, whereas $\operatorname{Tors}\left(\mathrm{B}_{3}^{\prime} / \mathrm{B}_{2}^{\prime}\right)=\mathbb{Z} / 4 \oplus \mathbb{Z} / 4 \oplus \mathbb{Z} / 2$ (as predicted by Lemma 4.8); hence, $\mathrm{B}[\alpha] \neq \mathrm{B}^{\prime}[\alpha]$ (cf. Remark 4.7).

Furthermore, $\bar{p}_{2}=\bar{p}_{3}=2$ and $\bar{q}=\bar{s}=1$, and in agreement with Lemma 4.8, we have Tors $\mathrm{B}_{2}=$ Tors $\mathrm{B}_{2}^{\prime}=\mathbb{Z} / 2 \oplus \mathbb{Z} / 2$. However, permuting the indices to $(0,2,1,3)$ (cf. Remark 4.9), we obtain a better bound: this time $\bar{p}_{2}=\bar{p}_{3}=\bar{q}=$ $\bar{s}=1$ and, hence, Tors $\mathrm{B}_{2}=$ Tors $\mathrm{B}_{2}^{\prime}=0$.

\section{REMARK 6.3}

This example also explains why, in the proof of Theorem 1.7(3) in Section 5, we had to consider the matrix (5.5) with rather long sequences of minors instead of a much simpler matrix given by Corollary 4.6: the latter would not work, as the corresponding module may have torsion.

\section{References}

[1] N. Aoki and T. Shioda, "Generators of the Néron-Severi group of a Fermat surface" in Arithmetic and Geometry, Vol. I, Progr. Math. 35, Birkhäuser Boston, Boston, 1983, 1-12. MR 0717587. DOI 10.1007/BF01589436. 
[2] A. Degtyarev, Lines generate the Picard groups of certain Fermat surfaces, J. Number Theory 147 (2015), 454-477. MR 3276333.

DOI 10.1016/j.jnt.2014.07.020.

[3] J. Delsarte, "Nombre de solutions des équations polynomiales sur un corps fini" in Séminaire Bourbaki, Vol. 1, no. 39, Soc. Math. France, Paris, 1995, 321-329. MR 1605138.

[4] V. V. Nikulin, Integer symmetric bilinear forms and some of their geometric applications, Izv. Akad. Nauk SSSR Ser. Mat. 43 (1979), no. 1, 111-177, 238; English translation in Math. USSR-Izv. 14 (1979), 103-167. MR 0525944.

[5] M. Schütt, T. Shioda, and R. van Luijk, Lines on Fermat surfaces, J. Number Theory 130 (2010), 1939-1963. MR 2653207. DOI 10.1016/j.jnt.2010.01.008.

[6] I. Shimada and N. Takahashi, Primitivity of sublattices generated by classes of curves on an algebraic surface, Comment. Math. Univ. St. Pauli 59 (2010), 77-95. MR 2789841.

[7] T. Shioda, On the Picard number of a Fermat surface, J. Fac. Sci. Univ. Tokyo Sect. IA Math. 28 (1981), 725-734. MR 0656049.

[8] - An explicit algorithm for computing the Picard number of certain algebraic surfaces, Amer. J. Math. 108 (1986), 415-432. MR 0833362. DOI $10.2307 / 2374678$.

Department of Mathematics, Bilkent University, Ankara, Turkey;

degt@fen.bilkent.edu.tr 